\title{
Trasplante de menisco: experiencia con seguimiento clínico y funcional a un año
}

\author{
Ortega-Orozco R, ${ }^{*}$ Rodríguez-Ávila NG, ${ }^{* *}$ Miranda-Ramírez E, *** Casillas-Arista KS, **** \\ Felipe-Gutiérrez $\mathrm{J},{ }^{* * * * *}$ Estrella-González CA, ${ }^{* * * * * *}$ Huante-Pineda $\mathrm{R} * * * * * * *$
}

Medyarthros, Medicina Deportiva y Artroscópica

RESUMEN. Introducción: En México se realizó el primer trasplante de menisco en 2002. El objetivo fue describir la evolución clínica de pacientes con dolor asociado al síndrome postmeniscectomía que fueron sometidos a trasplante de menisco alogénico (MAT), con bloques óseos vía artroscópica con seguimiento a un año. Material y métodos: Se realizó un estudio de cohorte retrospectiva con seguimiento a un año; se incluyeron pacientes con dolor en interlínea articular secundario a meniscectomía parcial o total, entre 15 y 55 años de edad, de ambos sexos, que fueron sometidos a MAT. La evolución clínica se evaluó con EVA, SF-36 y escala de Lysholm; los resultados se describen en frecuencias, porcentajes y valor de $p$ significativa $<0.05$. Resultados: Se incluyeron nueve pacientes; ocho hombres y una mujer con edad de $\mathbf{3 1 . 3}$ años (16-52), el seguimiento fue de 16.6 meses (12-23). La EVA prequirúrgica fue de 8.5 (6-10) y al año de seguimiento el promedio de esta escala fue de $\mathbf{1 . 2 5}$ $(0-5)(p<0.001)$. En el seguimiento a un año posterior al MAT, la escala de Lysholm fue en promedio de $91(68-100)$ y la SF-36 fue en promedio de 79.17 (65-92.92); tres pacientes regresaron a su práctica
ABSTRACT. Introduction: In Mexico, the first meniscus transplant was performed in 2002 . The objective was to describe the clinical evolution of patients with pain associated with postmenisectomy syndrome, who underwent meniscal allograft transplantation (MAT), with bony blocks via arthroscopy, with follow-up to one year. Material and methods: A retrospective cohort study with a one-year follow-up was conducted. We included patients with joint interlining pain secondary to partial or total menisectomy, between 15 and 55 years of age, of both sexes, who underwent MAT. The clinical evolution was evaluated with visual analogue scale (VAS), SF-36 and Lysholm scale; the results are described in frequencies, percentages and $p$ value was significant at $<0.05$. Results: We included nine patients; eight men and one woman with an age of 31.3 years (16$52)$, the follow-up was 16.6 months (12-23). The preoperative VAS was $8.5(6-10)$ and, at one year of follow-up, the average of this scale was $1.25(0-5)(\mathrm{p}$ $<0.001)$. In the follow-up one year after the MAT, the Lysholm scale was on average $91(68-100)$ and the SF-36 was on average $79.17(65-92.92)$; three

\section{Nivel de evidencia: IV (cohorte retrospectiva)}

\footnotetext{
* Especialista en Traumatología y Ortopedia, Subespecialista en Cirugía Artroscópica y Medicina del Deporte. Director General de Medyarthros, Medicina Deportiva y Artroscópica; Jefe del Servicio de Artroscopía y Medicina del Deporte, Hospital Civil de Guadalajara. Guadalajara, Jalisco, México.

** Médico Especialista en Ortopedia, Adiestramiento en Artroscopía, Benemérito Hospital Civil «Fray Antonio Alcalde». Guadalajara, Jalisco, México.

*** Especialista en Traumatología y Ortopedia, Especialista en Módulo de Rodilla en Medyarthros, Medicina Deportiva y Artroscópica. Guadalajara, Jalisco, México.

**** Especialista en Medicina de Rehabilitación, Directora de Medyarthros Rehab, Guadalajara, Jalisco, México.

***** Enfermero, Enfermero Quirúrgico de Medyarthros, Medicina Deportiva y Artroscópica. Guadalajara, Jalisco, México.

****** Licenciada en Enfermería, Enfermera Quirúrgica de Medyarthros, Medicina Deportiva y Artroscópica. Guadalajara, Jalisco, México.

******* Licenciatura en Cultura Física y Deportes, Licenciatura en Terapia Física, Entrenador personal en Medyarthros, Medicina Deportiva y Artroscópica. Guadalajara, Jalisco, México.
}

Dirección para correspondencia:

Navid G. Rodríguez-Ávila

La Ley Núm. 2726, Col. Circunvalación Vallarta, CP 44680, Guadalajara, Jalisco, México.

E-mail: dra.navid_ortotrauma@hotmail.com

Este artículo puede ser consultado en versión completa en http://www.medigraphic.com/actaortopedica 
deportiva previa a la lesión. Conclusión: Éste es el primer reporte en México del seguimiento a un año del MAT; se demostró mejoría clínica significativa $(p<0.001)$ con remisión del dolor en la rodilla con meniscectomía previa y en algunos casos el retorno a la práctica deportiva.

Palabras clave: Menisco, trasplante, evolución, cirugía.

\section{Introducción}

El menisco es una estructura importante en la distribución de la carga, amortiguación, lubricación, estabilidad, propiocepción y nutrición del cartílago articular de la rodilla; ${ }^{1,2}$ su pérdida reduce la superficie articular femorotibial y se produce disminución en el área de contacto, aumento en la presión de carga y degeneración temprana del cartílago articular. ${ }^{3,4,5,6}$ En la actualidad existen diferentes técnicas para preservar el menisco, aunque no todas las lesiones meniscales son reparables y elegibles para meniscectomía; además, este procedimiento puede complicarse con el síndrome postmeniscectomía: los pacientes presentan dolor y artrosis, por lo que el trasplante de menisco alogénico (MAT) se convierte en la opción terapéutica indicada. ${ }^{7}$ Milachowski et al. ${ }^{8}$ realizaron el primer trasplante meniscal en humanos en el Hospital Universitario de MunichGrosshadern. Posteriormente, Keene et al..$^{9}$ en 1987 efectuaron el primer trasplante meniscal por vía artroscópica.

El MAT tiene como objetivo restaurar la anatomía normal y biomecánica articular de la rodilla, así como limitar los efectos negativos de la ausencia del menisco en su compartimento. Algunos estudios han demostrado que la mejoría de la biomecánica articular se relaciona con el incremento del área de contacto y la disminución de la presión sobre la superficie articular.,10,11

Recientemente se publicó un metaanálisis que demostró la supervivencia en $85.8 \%$ del menisco medial y $89.2 \%$ del lateral en un período de 5-10 años. ${ }^{12}$

En México hace poco más de una década se realizó el primer trasplante de menisco ${ }^{13} \mathrm{y}$ actualmente este procedimiento se efectúa en varios centros hospitalarios; existen únicamente dos publicaciones de pacientes mexicanos relacionados con un reporte de caso ${ }^{14} \mathrm{y}$ un artículo de revisión. ${ }^{15}$ El objetivo de nuestro estudio fue describir la evolución clínica de pacientes con dolor en compartimento femorotibial asociado al síndrome postmeniscectomía, sometidos a trasplante de menisco alogénico, con bloques óseos en su inserción anterior y posterior vía artroscópica con seguimiento a un año.

\section{Material y métodos}

El diseño del estudio es de cohorte retrospectiva que se realizó de Marzo de 2016 a Febrero de 2018 en una clínica patients returned to their sports practice prior to the injury. Conclusion: This is the first report in Mexico that describes the one year follow-up of the MAT; significant clinical improvement was demonstrated $(p<0.001)$ with remission of pain in the knee with previous menisectomy and in some cases the return to sports practice.

Key words: Meniscus, transplant, evolution, surgery.

de rodilla; se incluyeron pacientes con dolor en la interlínea articular, secundario a meniscectomía parcial o total, entre 15 y 55 años de edad, de ambos sexos, que fueron sometidos a MAT con bloques óseos en su inserción. Los pacientes aceptaron participar y otorgaron el consentimiento verbal; el estudio fue aprobado por un comité de ética en investigación.

Las variables evaluadas fueron edad, sexo, peso, talla, índice de masa corporal (IMC), tipo de actividad deportiva previa a la lesión, rodilla afectada, compartimento, eventos quirúrgicos previos al MAT, intervalo de tiempo entre la lesión y el trasplante, localización de la lesión meniscal, procedimientos concomitantes y complicaciones. Los datos se registraron en un cuestionario en formato electrónico. Las escalas que se utilizaron para evaluar la evolución clínica fueron: escala visual análoga (EVA) para estimar el dolor antes y después de la intervención con las siguientes categorías: dolor leve de 0 a 3; dolor moderado de 4 a 6; y, dolor severo de 7 a 10. En la escala de Lysholm para evaluar la función de la rodilla, la puntuación se clasifica con función mala $(<65)$, regular $(65-83)$, buena (84-93) y excelente $(>$ 94).

La evaluación SF-36 se utilizó para conocer la calidad de vida y nivel de satisfacción con el tratamiento, a los 12 meses posteriores al procedimiento quirúrgico (MAT): la puntuación igual o $>50$ se definió como un mejor estado de salud. Los pacientes fueron interrogados mediante entrevista telefónica.

\section{Técnica quirúrgica}

Los trasplantes de menisco se realizaron con técnicas ya descritas en la literatura ${ }^{15}$ por el mismo cirujano. Se demostró con maniobras clínicas y resonancia magnética (RMN) la estabilidad de la rodilla con evidencia de ligamentos íntegros o reparación previa; en algunos casos se llevó a cabo un procedimiento concomitante con MAT: en la mayoría de los casos se efectuó plastía de LCA.

El aloinjerto que se utilizó consistió en meniscos congelados, no irradiados, de un banco de tejidos; las medidas del aloinjerto se determinaron con referencia a la medición del platillo tibial en la RMN de la rodilla contralateral. ${ }^{16,17}$ 
El procedimiento quirúrgico inició con el paciente en posición decúbito supino bajo bloqueo regional espinal, previa asepsia y antisepsia del miembro pélvico, sin isquemia, se colocaron campos estériles; se inició abordaje del portal anterolateral y bajo visión artroscópica, el portal anteromedial; se confirmó la ausencia del menisco y se realizó el túnel posterior con guía para la inserción del aloinjerto desde la metáfisis contralateral, preparado previamente con suturas y marcaje y se introdujo por el portal extendiendo un poco sus bordes, enseguida, se redujo y se insertó el bloque posterior del aloinjerto dentro del túnel; se fijó el cuerno posterior con técnica todo-adentro, el cuerpo dentro-fuera y el cuerno anterior fuera-adentro, se realizó túnel anterior y el bloque óseo anterior se introdujo en el mismo, las suturas transóseas se recuperaron y se anudaron; bajo visión artroscópica se verificó la reducción y fijación adecuadas.

El programa de rehabilitación funcional inició el mismo día de la cirugía, control de la inflamación con crioterapia con rangos de movimiento limitados $\left(0^{\circ}\right.$ a $\left.90^{\circ}\right)$, con uso de máquina de movimiento pasivo continuo y ejercicios isométricos, reeducación temprana del cuádriceps, marcha asistida con muletas sin apoyo en las dos primeras semanas postquirúrgicas; la descarga de peso se difirió hasta la semana seis: consecutivamente, se trabajó el fortalecimiento muscular del cuádriceps y acondicionamiento físico hasta los seis meses posterior al MAT; se inició entrenamiento deportivo específico con regreso al deporte después de nueve meses.

\section{Análisis estadístico}

Se utilizó un formato electrónico para el registro y análisis de la información (Microsoft Excel TM Seattle, WA. EUA). Los resultados se presentan en frecuencias y porcentajes: la $\mathrm{p}<0.05$ se consideró significativa.

Fuente del financiamiento: no se obtuvo financiamiento específico para realizar la investigación.

\section{Resultados}

Todos los pacientes sometidos a MAT durante el período de estudio y con seguimiento a un año; se incluyeron nueve casos (Tabla 1); ocho hombres y una mujer. La edad promedio fue de 31.3 (rango 16-52); el seguimiento posterior al trasplante meniscal alogénico fue de 16.6 meses (rango 1223). El intervalo de tiempo entre la lesión inicial y el MAT fue en promedio de 67.4 meses (rango 8-240); de acuerdo con el IMC seis pacientes presentaron sobrepeso con promedio de $26.8 \mathrm{~kg} / \mathrm{m}^{2}$ (rango de $25.2-29.5$ ) y tres pacientes con peso normal. El lado afectado con mayor frecuencia fue la rodilla derecha $(n=6,67 \%)$ en el compartimento medial $(n=7,78 \%)$. En ocho pacientes se realizaron, previo al MAT, los siguientes procedimientos quirúrgicos: plastía aislada de LCA $(\mathrm{n}=1,11 \%)$, plastía de LCA más sutura meniscal ( $\mathrm{n}=3,33 \%)$, plastía de LCA más meniscectomía parcial o total $(\mathrm{n}=3,33 \%)$, plastía de revisión de LCA (n $=1,11 \%)$, además, siete $(78 \%)$ pacientes refirieron el an- tecedente de artroscopía previa. Los procedimientos que se efectuaron de manera concomitante con el MAT fueron: plastía de LCA $(n=1,11 \%)$ y plastía de revisión $(n=2$, $22 \%$ ). Se realizó RMN posterior al MAT en cinco $(55 \%)$ pacientes; se observó integridad de la cápsula, sin evidencia de separación menisco-capsular o extrusión del mismo. Se presentaron complicaciones únicamente en un paciente con re-rotura de LCA y dolor en la interlínea articular medial (n $=1,11 \%$ ); fue reintervenido y se realizó plastía de revisión con autoinjerto S-G y se observó integridad del trasplante meniscal.

En la Tabla 2 se describen los resultados individuales de EVA, escala de Lysholm y SF-36 en 8 (89\%) de nueve pacientes incluidos en el estudio; un paciente no se localizó, por lo que no fue posible completar la evaluación con las escalas a un año. La EVA en el período prequirúrgico fue de 8.5 (rango 6-10), en siete (87.5\%) pacientes el dolor fue severo. A los 12 meses de seguimiento posterior al MAT, el dolor disminuyó significativamente $(\mathrm{p}<0.001)$; en promedio la escala fue de 1.25 (rango 0-5): en cuatro pacientes se confirmó la ausencia de dolor $(\mathrm{EVA}=0)$, tres pacientes con dolor leve $(E V A=1-2)$ y únicamente un paciente presentó dolor moderado $(E V A=5)$. La recuperación funcional de la rodilla, según la escala de Lysholm que se aplicó un año posterior al MAT, fue buena con un promedio de 91 (rango 68-100); en siete (87.5\%) pacientes la recuperación fue de buena a excelente; únicamente en un caso la función fue regular. Asimismo, se encontró mejor calidad de vida en nuestro grupo de estudio, según la evaluación SF-36, con un promedio de 79.17 (rango 65-92.92); dos pacientes hombres futbolistas amateurs y una paciente que practicaban triatlón amateur regresaron a su actividad deportiva.

\section{Discusión}

En nuestro estudio se demostró evolución clínica y funcional satisfactoria con mejoría significativa del dolor en pacientes con meniscectomía previa que recibieron un trasplante de menisco alogénico. Los pacientes con práctica deportiva, previa a la lesión, lograron retornar a la misma: con base en los resultados del estudio se demostró que el trasplante de menisco alogénico es el tratamiento de elección en paciente con dolor postmeniscectomía.

Se ha demostrado la eficacia del MAT para restaurar la anatomía normal y biomecánica de la articulación de la rodilla con recuperación clínica y funcional satisfactorias en pacientes con síndrome postmeniscectomía. Cole et al. ${ }^{7}$ demostraron mejoría estadísticamente significativa en la escala de EVA y encuestas estandarizadas en el seguimiento a dos años en 32 (84\%) de 38 pacientes; estos resultados son comparables con el presente estudio donde encontramos mejoría significativa del dolor con escala EVA prequirúrgica de 8.5 (rango 6-10) versus escala postquirúrgica a 12 meses de seguimiento de 1.25 (rango 0-5) $(\mathrm{p}<0.001)$. En relación con la escala de Lysholm en un estudio se reportó mejoría funcional en 63 trasplantes de menisco en 57 pacientes en el seguimiento a corto 
Tabla 1: Características de los pacientes con síndrome postmeniscectomía sometidos a MAT.

\begin{tabular}{|c|c|c|c|c|c|c|c|c|c|c|c|}
\hline Caso & Edad & Sexo & Peso & Talla & IMC & $\begin{array}{l}\text { Tiempo } \\
\text { transcurrido } \\
\text { entre lesión- } \\
\text { trasplante }\end{array}$ & Rodilla & $\begin{array}{l}\text { Comparti- } \\
\text { mento }\end{array}$ & $\begin{array}{c}\text { Eventos } \\
\text { quirúrgicos } \\
\text { previos al MAT }\end{array}$ & $\begin{array}{l}\text { Procedimiento } \\
\text { concomitante }\end{array}$ & $\begin{array}{c}\text { Tiempo de } \\
\text { seguimiento } \\
\text { postrasplante }\end{array}$ \\
\hline 1 & 19 años & $\mathrm{H}$ & $76 \mathrm{~kg}$ & $1.78 \mathrm{~m}$ & $24 \mathrm{~kg} / \mathrm{m}^{2}$ & 10 meses & Izquierda & Medial & $\begin{array}{l}\text { - Plastía de LCA } \\
\text { - Artroscopía }\end{array}$ & Ninguno & 23 meses \\
\hline 2 & 38 años & $\mathrm{H}$ & $84 \mathrm{~kg}$ & $1.82 \mathrm{~m}$ & $25.4 \mathrm{~kg} / \mathrm{m}^{2}$ & 8 meses & Izquierda & Medial & $\begin{array}{l}\text { - Artroscopía } \\
\text { - Artroscopía } \\
\text { secundario } \\
\text { - Artrofibrosis }\end{array}$ & Ninguno & 20 meses \\
\hline 3 & 16 años & $\mathrm{H}$ & $101 \mathrm{~kg}$ & $1.85 \mathrm{~m}$ & $29.5 \mathrm{~kg} / \mathrm{m}^{2}$ & 28 meses & Derecha & Medial & $\begin{array}{l}\text { - Plastía de LCA } \\
\text { con aloinjerto + } \\
\text { sutura meniscal } \\
\text { - Artroscopía }\end{array}$ & $\begin{array}{l}\text { Plastía de } \\
\text { revisión de } \\
\text { LCA }\end{array}$ & 18 meses \\
\hline 4 & 46 años & $\mathrm{H}$ & $89 \mathrm{~kg}$ & $1.88 \mathrm{~m}$ & $25.2 \mathrm{~kg} / \mathrm{m}^{2}$ & 20 años & Derecha & Medial & Ninguno & $\begin{array}{l}\text { - Plastía LCA } \\
\text { - Plastía de } \\
\text { revisión LCA } \\
28 / 04 / 2017 \\
\text { (2nd look } \\
\text { MAT íntegro)* }\end{array}$ & 16 meses \\
\hline 5 & 44 años & $\mathrm{H}$ & $90 \mathrm{~kg}$ & $1.77 \mathrm{~m}$ & $28.7 \mathrm{~kg} / \mathrm{m}^{2}$ & 12 años & Derecha & Medial & $\begin{array}{l}\text { - Plastía LCA + } \\
\text { sutura meniscal } \\
\text { - Meniscectomía }\end{array}$ & Ninguno & 16 meses \\
\hline 6 & 25 años & $\mathrm{H}$ & $80 \mathrm{~kg}$ & $1.78 \mathrm{~m}$ & $25.2 \mathrm{~kg} / \mathrm{m}^{2}$ & 26 meses & Izquierda & Lateral & $\begin{array}{l}\text { - Plastía de LCA y } \\
\text { meniscectomía } \\
\text { - Artroscopía }\end{array}$ & Ninguno & 15 meses \\
\hline 7 & 26 años & $\mathrm{H}$ & $73 \mathrm{~kg}$ & $1.65 \mathrm{~m}$ & $26.8 \mathrm{~kg} / \mathrm{m}^{2}$ & 15 meses & Derecha & Medial & $\begin{array}{l}\text { - Plastía de LCA } \\
\text { + sutura } \\
\text { meniscal } \\
\text { - Meniscectomía } \\
\text { total }\end{array}$ & Ninguno & 15 meses \\
\hline 8 & 52 años & M & $61 \mathrm{~kg}$ & $1.64 \mathrm{~m}$ & $22.7 \mathrm{~kg} / \mathrm{m}^{2}$ & 10 años & Derecha & Medial & $\begin{array}{l}\text { - Plastía de LCA } \\
\text { aloinjerto y } \\
\text { meniscectomía } \\
\text { - Plastía de } \\
\text { revisión con } \\
\text { autoinjerto }\end{array}$ & Ninguno & 14 meses \\
\hline 9 & 17 años & $\mathrm{H}$ & $64 \mathrm{~kg}$ & $1.70 \mathrm{~m}$ & $22.1 \mathrm{~kg} / \mathrm{m}^{2}$ & 16 meses & Derecha & Lateral & $\begin{array}{l}\text { - Plastía de LCA } \\
\text { + meniscectomía } \\
\text { - Artroscopía }\end{array}$ & $\begin{array}{l}\text { Plastía de } \\
\text { revisión de } \\
\text { LCA }\end{array}$ & 12 meses \\
\hline
\end{tabular}

plazo $(79 \pm 19$ a $3.1 \pm 1.5$ años $) ;{ }^{18}$ sin embargo, a largo plazo (13.5 años) ocurrió deterioro parcial de la función. En nuestro estudio con seguimiento a corto plazo (rango 12-23 meses) la recuperación en siete $(87.5 \%)$ pacientes fue buena o excelente con promedio de 91 (rango 68-100); únicamente en un caso la función fue regular. Aun cuando la mejoría a largo plazo no se mantiene en los niveles altos alcanzados en el corto plazo, el MAT es una buena opción de tratamiento para retardar el desarrollo de osteoartrosis degenerativa en pacientes jóvenes con síndrome postmeniscectomía y retrasar el momento del reemplazo articular total de la rodilla. ${ }^{12,18,19}$

Se recomienda realizar el MAT de manera temprana para obtener mejores resultados en la calidad de vida. Sekiya et al. ${ }^{20}$ evaluaron 25 pacientes con edad promedio de 30 años; se demostró un mejor estado de salud posterior al procedimiento quirúrgico con el cuestionario SF-36 (resultado pro- medio 71.95) que se relacionó con menor tiempo transcurrido (67 meses) entre la lesión inicial y el MAT; en contraste, en el estudio que nos ocupa el SF-36 fue de 79.17 (rango 65-92.92) y no encontramos diferencia en la evolución clínica y funcional respecto al intervalo entre la lesión y cirugía.

El primer trasplante de menisco con aloinjerto en México se realizó en el año $2002 ;{ }^{13}$ recientemente, se publicó una serie de 35 pacientes mexicanos con edad promedio de 32.4 años (rango 19-50 años) con MAT vía artroscópica en un período de siete años: ${ }^{15}$ todos los procedimientos fueron efectuados por el mismo cirujano. Se reportó mejoría significativa del dolor y la función sin precisar el tiempo de seguimiento, no se describieron las escalas aplicadas antes y después del trasplante. El presente estudio es el primer reporte en México con seguimiento igual o mayor a un año posterior al MAT; se observó mejoría clínica significativa del dolor $(\mathrm{p}<0.001)$ con 


\begin{tabular}{|c|c|c|c|c|}
\hline \multicolumn{5}{|c|}{$\begin{array}{l}\text { Tabla 2: Evaluación del dolor con EVA, recuperación } \\
\text { funcional de la rodilla (escala Lysholm) y calidad de vida (SF-36) } \\
\text { en pacientes sometidos a MAT con seguimiento a } 12 \text { meses. }\end{array}$} \\
\hline Caso & $\begin{array}{c}\text { EVA: previo } \\
\text { a MAT }\end{array}$ & $\begin{array}{l}\text { EVA: } 12 \\
\text { meses } \\
\text { post-MAT* }\end{array}$ & $\begin{array}{l}\text { Escala } \\
\text { Lysholm:** } \\
12 \text { meses } \\
\text { post-MAT }\end{array}$ & $\begin{array}{l}\text { SF-36:** } \\
12 \text { meses } \\
\text { post-MAT }\end{array}$ \\
\hline 1 & 8 & 2 & 90 & 77.91 \\
\hline 2 & 7 & 0 & 100 & 81.20 \\
\hline 3 & 8 & 0 & 95 & 92.92 \\
\hline 4 & 10 & 5 & 68 & 65.00 \\
\hline 5 & ND & ND & ND & ND \\
\hline 6 & 9 & 0 & 100 & 80.14 \\
\hline 7 & 6 & 0 & 95 & 69.02 \\
\hline 8 & 10 & 2 & 85 & 89.86 \\
\hline 9 & 10 & 1 & 95 & 77.36 \\
\hline
\end{tabular}

escalas estandarizadas. Es necesario realizar estudios a largo plazo, 10-15 años, con mayor número de pacientes y diseño prospectivo (nivel de evidencia I-II) para determinar si la mejoría clínica y funcional con el trasplante alogénico persiste a largo plazo en pacientes mexicanos.

La principal fortaleza de este estudio se relaciona con la técnica quirúrgica estándar de aloinjerto con bloques óseos en su inserción anterior y posterior por vía artroscópica, realizada por el mismo cirujano; en contraste, se identifican varias debilidades, como el diseño retrospectivo que limita la disponibilidad de datos para la comparación pre y postquirúrgica, el número de pacientes incluidos y el período de seguimiento a corto plazo.

En conclusión, en nuestro estudio se describe la evolución clínica en pacientes con meniscectomía previa que recibieron trasplante de menisco alogénico y seguimiento igual o mayor a un año; se demostró mejoría clínica significativa con remisión del dolor. El trasplante meniscal es un procedimiento efectivo para lograr la recuperación clínica y funcional de pacientes con síndrome de postmeniscectomía; y un procedimiento concomitante en la reconstrucción de LCA de revisión y la reparación del cartílago articular en un compartimento con menisco deficiente.

\section{Agradecimientos}

Los autores agradecen a la Dra. Ana María Contreras de la Unidad de Investigación y Desarrollo Tecnológico (UNIDET) de Medyarthros por su asesoría en la redacción de este artículo.
Bibliografía

1. Makris EA, Hadidi P, Athanasiou KA. The knee meniscus: structurefunction, pathophysiology, current repair techniques, and prospects for regeneration. Biomaterials. 2011; 32(30): 7411-31.

2. Rosso F, Bisicchia S, Bonasia DE, Amendola A. Meniscal allograft transplantation: a systematic review. Am J Sports Med. 2015; 43(4): 998-1007.

3. Dandy DJ, Jackson RW. Meniscectomy and chondromalacia of the femoral condyle. J Bone Joint Surg Am. 1975; 57(8): 1116-9.

4. Fairbank TJ. Knee joint changes after meniscectomy. J Bone Joint Surg Br. 1948; 30B(4): 664-70.

5. Johnson RJ, Kettelkamp DB, Clark W, Leaverton P. Factors effecting late results after meniscectomy. J Bone Joint Surg Am. 1974; 56(4): 719-29.

6. Outerbridge RE. The etiology of chondromalacia patellae. $J$ Bone Joint Surg Br. 1961; 43-B: 752-7.

7. Cole BJ, Dennis MG, Lee SJ, Nho SJ, Kalsi RS, Hayden JK, et al. Prospective evaluation of allograft meniscus transplantation: a minimum 2-year follow-up. Am J Sports Med. 2006; 34(6): 919-27.

8. Milachowski KA, Weismeier K, Wirth CJ. Homologous meniscus transplantation. Experimental and clinical results. Int Orthop. 1989; 13(1): 1-11.

9. Keene GC, Paterson RS, Teague DC. Advances in arthroscopic surgery. Clin Orthop Relat Res. 1987; (224): 64-70.

10. Alhalki MM, Hull ML, Howell SM. Contact mechanics of the medial tibial plateau after implantation of a medial meniscal allograft. A human cadaveric study. Am J Sports Med. 2000; 28(3): 370-6.

11. Paletta GA Jr, Manning T, Snell E, Parker R, Bergfeld J. The effect of allograft meniscal replacement on intraarticular contact area and pressures in the human knee. A biomechanical study. Am J Sports Med. 1997; 25(5): 692-8.

12. Bin SI, Nha KW, Cheong JY, Shin YS. Midterm and long-term results of medial versus lateral meniscal allograft transplantation: a metaanalysis. Am J Sports Med. 2018; 46(5): 1243-1250.

13. Berumen-Nafarrate E. Una historia que vale la pena contarse. Carta al editor. Acta Ortop Mex. 2002; 16(4): 241.

14. Camacho-Franco L, Tarazona-Velutini P, Montalvo-Galindo M, Rivera-Ramírez A, Zamora-Muñoz P, Testas-Hermo M. Trasplante meniscal. Reporte de un caso con ocho meses de seguimiento. Acta Ortop Mex. 2005; 19(2): 75-9.

15. Cruz-Lopez F, Olivos-Meza A, Llano-Rodríguez LT, Ibarra C. Arthroscopic meniscal transplantation technique. J Sports Med Doping Stud. 2015; 6: 171.

16. Getgood A, LaPrade RF, Verdonk P, Gersoff W, Cole B, Spalding T; IMREF Group. International meniscus reconstruction experts forum (IMREF) 2015 consensus statement on the practice of meniscal allograft transplantation. Am J Sports Med. 2017; 45(5): 1195-1205.

17. Yoon JR, Jeong HI, Seo MJ, Jang KM, Oh SR, Song S, et al. The use of contralateral knee magnetic resonance imaging to predict meniscal size during meniscal allograft transplantation. Arthroscopy. 2014; 30(10): 1287-93.

18. van der Wal RJ, Thomassen BJ, van Arkel ER. Long-term clinical outcome of open meniscal allograft transplantation. Am J Sports Med. 2009; 37(11): 2134-9.

19. Hommen JP, Applegate GR, Del Pizzo W. Meniscus allograft transplantation: ten-year results of cryopreserved allografts. Arthroscopy. 2007; 23(4): 388-93.

20. Sekiya JK, West RV, Groff YJ, Irrgang JJ, Fu FH, Harner CD. Clinical outcomes following isolated lateral meniscal allograft transplantation. Arthroscopy. 2006; 22(7): 771-80. 